\title{
Wide field optical surveys
}

\author{
Naoki Yasuda \\ National Astronomical Observatory, Tokyo, Japan \\ email: naoki.yasuda@nao.ac.jp
}

The products of optical wide-field survey are very valuable for their own purposes like the studies of large scale structure of galaxies, evolution of galaxies, Galactic structure and so on. At the same time optical view of sky will provide basic reference for the observation at other wavelength ranges. For this reason Palomar all sky survey has been used for various astronomical studies over 50 years. Now in the era of electronic devices, digital archives, and powerful computer systems, modern observation will replace the Palomar all sky survey.

One of the pioneering work in this field is the Sloan Digital Sky Survey (SDSS) which has been carried out during 2000-2005. SDSS gives us the five band images over 8,000 square degrees of the sky, nearly 200 milliion celestial objects detected, and spectra of more than 675000 galaxies, 90000 quasars, and 185000 stars. These huge and wellcalibrated datasets of SDSS has been a good material for the development of Virtual Observatory (VO) and the demonstration of the effectiveness of VO. We will introduce the scientific results which were obtained by the large SDSS database. From these scientific studies we can learn about how VOs should be and what functionality VOs need.

We also discuss about the different types of optical surveys, i.e., deep surveys and time transient surveys. In order to reveal the structure and evolution of galaxies at the early Universe, a number of deep surveys have been carried out. As an example, we will discuss the results of deep surveys done by Subaru telescope.

On the other hand, time transient surveys give us a different aspect of astronomy. Many distant supernovae are discovered recently from time transient surveys. These supernovae are used for the investigation of the nature of dark energy. These different kinds of surveys also give us some insight for VOs. 